\title{
Petroleum Hydrocarbon Spills in the Environment and Abundance of Microbial Community Capable of Biosurfactant Production
}

Chirwa EMN* and Bezza FA

Water Utilisation and Environmental Engineering Division, Department of Chemical Engineering, University of Pretoria, Pretoria 0002, Republic of South Africa

\begin{abstract}
Petroleum industries generate wastes, some of which may be considered hazardous because of the presence of toxic organics and heavy metals. The wastes can bioaccumulate in food chains where they disrupt biochemical or physiological activities of many organisms, thus causing carcinogenesis of some organs, mutagenesis in the genetic material, impairment in reproductive capacity. Bioremediation constitutes the primary mechanism for the elimination of hydrocarbons from contaminated sites by natural existing populations of microorganisms. In this work, microbial community composition and metabolic potential have been explored in petroleum-hydrocarbon contaminated wood treatment plant soil. A collection of strains, adapted to grow on minimal medium supplemented with coal tar creosote, was obtained and the diversity of the bacterial collection was analysed by 16S rRNA gene-based 454 pyrosequencing. Sequencing of the bands revealed a high proportion of Proteobacteria represented by the Alpha, Beta and Gamma subclasses, suggesting that Proteobacteria and especially Gamma subclass is a dominant group in coal tar creosote contaminated soils. The biotechnological potential of the operational taxonomic units (OUTs) revealed a significant degradation of creosote PAHs and production of biosurfactant with important emulsification activities during the bioremediation process.
\end{abstract}

Keywords: Biosurfactant; Bioremediation; Hydrocarbonoclastic Bacteria; Petroleum Hydrocarbons

\section{Introduction}

A considerable amount of oily sludge can be generated from the petroleum industry during its crude oil exploration, production, transportation, storage, and refining processes [1]. In particular, the sludge generated during the petroleum refining process has received increasing attention in recent years. It contains a high concentration of petroleum hydrocarbons (PHCs) and other recalcitrant components. As being recognized as a hazardous waste in many countries, the improper disposal or insufficient treatment of oily sludge can pose serious threats to the environment and human health [2]. Short et al. [3] reported that thirteen years after the Exxon Valdez oil spill in Prince William Sound, the toxic effects are still being felt due to the remaining bulk of the less-weathered subsurface oil. A random sampling of underground fuel storage tanks conducted by U.S. Environmental Protection Agency (USEPA) in the United States revealed about 35\% leaks in these tanks $[4,5]$.

Due to a lot of factors, oil pollution has continued to be of great concern to the entire world. One of the main issues faced by refineries and petrochemical industries is related to the safe disposal of this residue, since its destination and/or inappropriate treatment can cause serious impact to the environment and potential risk to human health [6]. Petroleum hydrocarbons are organic pollutants of major concern due to their wide distribution, persistence, complex composition, and toxicity. They can bioaccumulate in food chains where they disrupt biochemical or physiological activities of many organisms, thus causing carcinogenesis of some organs, mutagenesis in the genetic material, impairment in reproductive capacity [5]. The most common petroleum hydrocarbons include aliphatic, branched, and cycloaliphatic alkanes, as well as monocyclic and polycyclic aromatic hydrocarbons (PAHs). PAHs include naphthalene, fluorene, phenanthrene, anthracene, fluoranthene, pyrene, benzo[a]anthracene, benzo[a]pyrene. Combined cycloaliphatic-aromatic structures can also be found in crude oil. Each petroleum fraction is usually composed of hundreds of different hydrocarbon molecules rather than a defined composition. Thus, fractions are dissimilar in terms of volatility, bioavailability, toxicity, degradability, and persistence. This complex array of compounds depicts the tremendous challenge for designing effective bioremediation strategies, which can be illustrated by the effects of major contamination events in the past $[7,8]$.

Various physicochemical methods are available for the treatment of petroleum waste, although many of these technologies are costly, energy intensive, inefficient and not eco-friendly. Bioremediation technology on the other hand, which is based on natural microbial population of contaminated sites has been recognized as a sustainable, economic, environmentally friendly and versatile alternative clean-up strategy [9]. The success of bioremediation technologies applied to hydrocarbonpolluted environments highly depends on the biodegrading capabilities of native microbial populations or exogenous microorganisms used as inoculants [10]. The communities which were exposed to hydrocarbons become adapted, exhibiting selective enrichment and genetic changes [11]. The adapted microbial communities can respond to the presence of hydrocarbon pollutants within hours [11] and exhibit higher biodegradation rates than communities with no history of hydrocarbon contamination [12].

The major issue hindering rapid degradation of the petroleum sludge in biological method is the poor availability of hydrocarbons to the microorganisms due to their complexity and water insoluble nature.

*Corresponding author: Chirwa EMN, Water Utilisation and Environmental Engineering Division, Department of Chemical Engineering, University of Pretoria, Pretoria 0002, Republic of South Africa, Tel: +27-12-420 5894; E-mail: Evans.Chirwa@up.ac.za

Received May 31, 2015; Accepted July 08, 2015; Published July 18, 2015

Citation: Chirwa EMN, Bezza FA (2015) Petroleum Hydrocarbon Spills in the Environment and Abundance of Microbial Community Capable of Biosurfactant Production. J Pet Environ Biotechnol 6: 237. doi:10.4172/2157-7463.1000237

Copyright: (C) 2015 Chirwa EMN, et al. This is an open-access article distributed under the terms of the Creative Commons Attribution License, which permits unrestricted use, distribution, and reproduction in any medium, provided the original author and source are credited. 
Hence, the greatest challenge for microbiologists and bioengineers in the area of petroleum sludge bioremediation is developing the technology that will ensure the interaction of microorganisms with the complex hydrocarbon overcoming the pollutant complexity and insolubility $[13,14]$

Biosurfactants play a pivotal role in the biodegradation of hydrophobic aromatic compounds contained in petroleum and heavy oil, but the extent to which various petroleum-degrading microorganisms produce these substances is still unknown [15]. Since long term exposure to petroleum hydrocarbons would be expected to select for the development of biosurfactant-producing bacteria via horizontal gene transfer and metabolic switching [16], chronically contaminated sites should contain bacteria that produce effective surfactants that can be used by many different petroleum-degrading species that are indigenous to petroleum-dominated habitats [15].

In our previous study of creosote contaminated soil bioremediation (Bezza and Chirwa, 2015, in press) microbial consortia was enriched from the wood treatment plant soil using Creosote as a source of carbon and energy. The soil sample was collected from the top surface layer $(15 \mathrm{~cm})$ at a wood impregnation plant in the outskirts of Pretoria (Gauteng, South Africa). The sampling site is contaminated by a variety of industrial waste products related to Creosote processing that have been released over the last twenty years. The soil contained about 21 percent by weight TOC and $1.5 \%$ of PAH (such as naphthalene, fluorene, phenanthrene, anthracene, fluoranthene, pyrene, benzo[a] anthracene, benzo[a]pyrene). The bioremediation technique proved efficient in the removal of PAHs in the contaminated soil through supplying oxygen and nutrients. An examination of the waste residue after 45 days of treatment revealed a $79 \%$ PAH removals in the nutrient amended bioslurry reactors. PAH percent removals varied from 52.0 percent for 5 and 6 ring PAHs to 82.0 percent for 2 and 3 ring PAHs in nutrient amended samples where the community dynamics was studied. The evaluated process was aerobic thus it supported an array of aerobic bacteria. The objective of this work was to assess the microbial community composition and abundance of Hydrocarbonoclastic and biosurfactant producing bacteria from the creosote contaminated soil using high-resolution $16 \mathrm{~S}$ rRNA tag pyrosequencing technique as described by Albers et al. [17]. The abundance of different Hydrocarbonoclastic bacteria was assessed in the creosote contaminated soil samples before the inoculation and after the bioremediation of the soil samples.

\section{Materials and Methods}

\section{Sample collection}

Creosote degrading microbial cultures were enriched from soil obtained from wood treatment plant in Pretoria West (Pretoria, South Africa). Due to the creosote contamination at the site, high levels of PAHs, PCBs and other petrochemical organic contaminants were detected in the soil. Microbial inocula were prepared by shaking $5 \mathrm{~g}$ (wet weight) of the inoculum source in $100 \mathrm{ml}$ of MSM [18] containing 5\% $(\mathrm{v} / \mathrm{v})$ creosote as a source of carbon and energy. Microbial cultures for the pyrolysis analysis were sampled by withdrawing $5 \mathrm{ml}$ liquid culture from the shake flask before the enrichment. The enrichment culture was grown for 7 days under continuous shaking at $120 \mathrm{rpm}$ in a Labcon SPL-MP 15 Orbital Shaker (Labcon Laboratory Services, South Africa). Aliquots of enrichment cultures $(5 \mathrm{ml})$ were aseptically transferred to fresh $5 \%(\mathrm{v} / \mathrm{v})$ creosote containing medium and incubation continued. This enrichment procedure was repeated for five successive transfers.

\section{Biosliurrty reactors and sampling procedure}

The $2 \mathrm{~L}$ bioslurry reactors sampled were set up with the contaminated soil to give 30 percent soil-water slurry. The reactors were seeded with the enriched microbial cultures and sufficient nutrients $\mathrm{N}$ and $\mathrm{P}$ were added to obtain a C:N:P ratio of 100:10:1 [19] to satisfy the demands of the microbes degrading the petroleum hydrocarbon contaminants. The reactors were vigorously mixed using overhead mechanical mixers and run in triplicates at $37^{\circ} \mathrm{C}$ for 45 days. After the 45 days of bioremediation samples were collected for microbial dynamics study. The reactors were sampled by withdrawing slurry material from each reactor. The samples for the microbial analysis were collected in sterile $15 \mathrm{ml}$ plastic bottles. During transportation to the laboratory, the $15-\mathrm{ml}$ samples were frozen immediately in a cooler with dry ice. The samples were stored in the laboratory at $20^{\circ} \mathrm{C}$.

\section{DNA extractions}

To extract DNA from samples, frozen sample filters were removed from the freezer and immediately crushed into small pieces in the tube by using a sterile spatula. The frozen filter pieces were added to a tube containing a bead-beating matrix and buffers according to the standard protocol for the Fast DNA spin kit for soil (MP Biomedicals, Solon, $\mathrm{OH}$ ). DNA extractions were further carried out according to the manufacturers' instructions. The amount and quality of the extracted DNA were estimated on $1 \%$ agarose gels and using Nano Drop spectrophotometer readings (Thermo Scientific, Wilmington, DE). Extracted DNA was stored at $-20^{\circ} \mathrm{C}$ for downstream processing.

\section{PCR amplification and pyrosequencing}

Samples were prepared for 454 pyrosequencing using two-step PCR [20]. Initial PCR Mastermix was $1 \times$ Phusion HF buffer (with $\mathrm{MgCl}_{2}$; Finnzymes Oy, Espoo, Finland), $0.2 \mathrm{mM}$ of dNTP mixture, 0.5 $\mathrm{U}$ Phusion Hot Start DNA polymerase (Finnzymes), $0.5 \mu \mathrm{M}$ of each primer, $1 \mu \mathrm{l}$ of template, and sterile Milli-Q water to a final volume of $25 \mu$ l. Primers were MPRK341F (5'CCTAYGGGRBGCASCAG-3') and MPRK806R (5'GGACTACNNGGGTATCTAAT-3'), slightly modified from $\mathrm{Yu}$ et al. [21]. The primer set targets the 16S rRNA genes flanking the V3 and V4 regions with an overall coverage of $85 \%$ and $80 \%$ for bacteria and archaea, respectively. PCR conditions were an initial denaturation step of $98^{\circ} \mathrm{C}$ for $30 \mathrm{~s}$, followed by 30 cycles of denaturation at $98^{\circ} \mathrm{C}$ for $5 \mathrm{~s}$, annealing at $5^{\circ} \mathrm{C}$ for $20 \mathrm{~s}$, elongation at $72^{\circ} \mathrm{C}$ for $20 \mathrm{~s}$, and a final extension step of $72^{\circ} \mathrm{C}$ for $5 \mathrm{~min}$. Immediately before running on $1 \%$ agarose gels with ethidium bromide for UV visualization, PCR products were incubated at $7^{\circ} \mathrm{C}$ for $3 \mathrm{~min}$ and then transferred to ice. The bands of PCR products were cut from the gels and purified using Montage DNA Gel Extraction kits (Millipore, Bedford, MA).

To add adaptor and tags to the PCR products, we performed a second round of PCR using DNA fragments from the purified bands as templates. The second PCR amplification was performed as described above, except that we used the primers MPRK341F and MPRK806R with adaptors and 22 barcodes of 10 nucleotides length (on the forward primer). Further, the number of cycles for denaturation, annealing, and elongation was reduced to 20 . The PCR products were processed, run on agarose gels, and purified as described above

Amplified fragments with adapters and tags were quantified using a Qubit fluorometer (Invitrogen, Life technologies, Carlsbad, CA) and mixed in equal concentrations $\left(10^{8}\right.$ copies/ $\left.\mu l\right)$ to ensure equal representation of each sample. A 454 sequencing run was performed on a 70_75 GS Pico Titer Plate using a GS FLX pyrosequencing system according to the manufacturer's instructions (Roche, Mannheim, 
Germany). Sorting and trimming of sequences $>150$ bp was done by the Pipeline Initial Process (http://rdp.cme.msu.edu) as previously described $[17,22]$.

\section{Bioinformatic analyses}

Sequence processing was performed in Mothur [23]. In Mothur, poor quality sequences were set as sequences with a length less than 550 bases, contained ambiguous bases and homopolymers greater than 6 bases or did not have a barcode and a primer sequence. Multiple sequence alignments were performed using the program MAFFT, version 6.925 [24], with the E-INS-i strategy assuming multiple conserved regions and long gaps. After subsequent preclustering to an alignment of known $16 \mathrm{~S}$ bacterial sequences, chimeras check with UCHIME [25], the sequences were aligned and clustered into operational taxonomic units (OTUs) using the furthest neighbour algorithm with $97 \%$ similarity threshold. Bacterial data were summarized at phylum, class, order, family and genus and species levels (Figure 1). These OTUs were taxonomically identified by the RDP-II Naïve Bayesian Classifier [26] using an $80 \%$ confidence threshold.

\section{Results and Discussion}

\section{Distribution and abundance of bacterial groups}

Using a similarity threshold of $97 \%$ to cluster sequences within the same operational taxonomic units (OTUs), a total of 320 OUTs were found from creosote contaminated soil before the bioremediation was conducted (ENG A) and 53 OUTs were obtained in the sample after the bioremediation treatment (ENGB), (Figures 1 and 2a). The overall distribution of the main prokaryotic groups (phyla or classes) showed a dominance of sequences within, Gammaproteobacteria, Alphaproteobacteria, Bacilli, Betaprotobacteria and, Acidobacteria (Supplementary Figure 1). At the phylum level, the foremost populations in the ENGA were Proteobacreia whereas and ENGB reactors included Proteobacreia, Firmicutes, Actinobacteria, Acidobacteria at $1 \%$ cut off. Proteobacteria was the dominant bacterial phylum, representing $99 \%$ of the $16 \mathrm{~S}$ rDNA reads from the 320 most abundant bacterial OTUs in sample A at $1 \%$ cut off (Supplementary Figure 1; Relative abandance). Pseudomonadaceae (94\%) was the most dominant family from the sample whereas Enterobacteriaceae $(0.018 \%)$ and Xanthomonadaceae $(0.0026 \%)$ were observed at $1 \%$ cut off level (Figure 1; Relative abandance). Proteobacteria represented $88.8 \%$ of the phyla in the ENGB whereas Firmicutes (2.22\%), Actinobacteria (1.6\%),

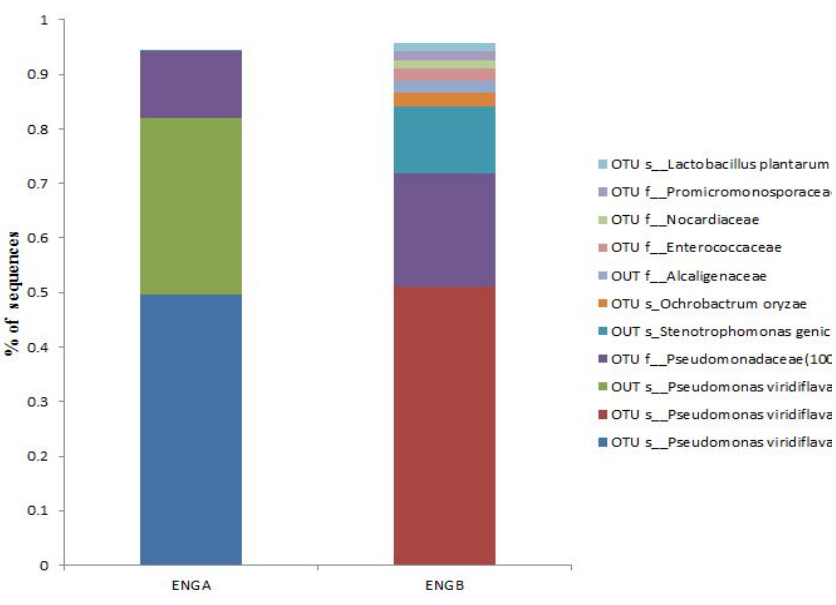

Figure 1: The overall distribution of the prokaryotic groups.
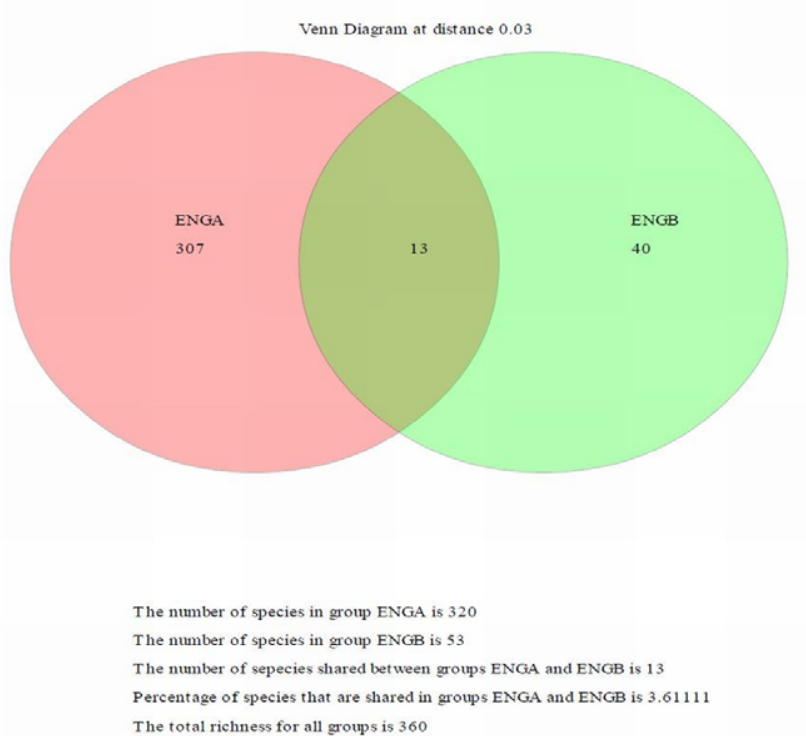

Figure 2a:Operational taxonomic unitsobtained after bioremediation treatment.

Acidobacteria (1.6\%). Proteobacteria have been identified in many studies as the predominant phylum in soil samples $[27,28]$ playing an integral role in nutrient cycling [28].The Proteobacteria encompass enormous morphological, physiological and metabolic diversity, and are of great importance to global carbon, nitrogen and sulphur cycles [27].

Bacteria in the phyla Proteobacteria,Actinobacteria, Firmicutes, Bacteroidetes and Chlamydiae have been reported as hydrocarbon degraders [29]. The current study showed that besides the above mentioned phyla Verrucomicrobia; Chloroflexi; Planctomycetes; WPS-2; Chloroflexi; Armatimonadetes; Gemmatimonadetes; WPS2; TM7 bacterial phyla were observed in the creosote contaminated soil. Our results are in agreement with most studies showing the importance of the Proteobacteria, especially the Gamma division, in hydrocarbon-polluted soil microbial communities or natural asphalts [27,30]. Shared families in both samples were Enterobacteriaceae; Pseudomonadaceae; Xanthomonadaceae; Micrococcaceae; Brucellaceae; Bradyrhizobiaceae; Sphingomonadaceae; Bacillaceae; Comamonadaceae; Methylobacteriaceae; Oxalobacteraceae and Weeksellaceae. Among the 13 bacterial OTUs shared between A and B (Figure 1; Relative abundance) Pseudomonadaceae is the dominant family. Strains affiliated with the gamma-proteobacteria group are associated with members of the genera Pseudomonas, Stenotrophomonas, which have been reported as being hydrocarbonoclastic strains [16,31]. The term "hydrocarbonoclastic" has been used to describe hydrocarbon utilizing microorganisms. This specifically relates to microbes that are capable of degrading hydrocarbons, and all of which share some characteristics like having a capable and efficient hydrocarbon uptake system, have receptor sites for binding hydrocarbons and are capable of producing surfactants [32].

Most of the shared OTUs belong to the genera Pseudomonas, Stenotrophomonas, Ochrobactrum, Achromobacter, Enterococcus, Cellulosimicrobium, Lactobacillus, Brevibacillus, Ornithinibacillus, Arthrobacter, Paenibacillus (Figure 1; Shared OTUs). Species from these genera are reported as efficient biosurfactant producers [3338]. The community in sample ENGB was dominated by members 


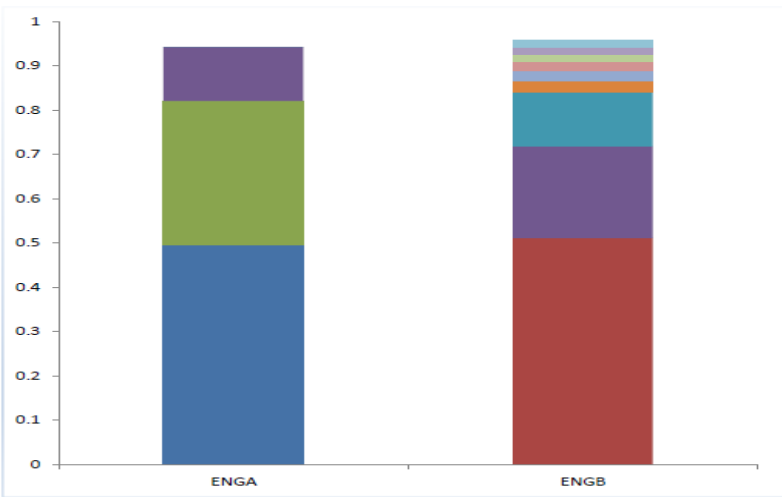

Figure 2b: Relative abundance of the microbial community before the inoculation of the enriched consortium (ENGA) and after 40 days ofbioremediation of the creosote contaminated soil (ENGB).

of the Enterobacteriaceae family (Figure 2a) representative genera in this family like Klebsiella and Serratia were reported as efficient biosurfactant producers and capable of hydrocarbon degradation [39,40]. Pseudomonas spp which are dominant in sample A (Figure 2b) are able to endure and metabolize contaminants that are considered very toxic to other bacteria. Pseudomonads have a huge potential for bioremediation, several studies have proved that Pseudomonas sp. can utilize a vast range of contaminants either naturally present or xenobiotic [41]. Pseudomonas sp. is a prolific producer of a number of biosurfactants and extra cellular enzymes (like lipase). Importantly, these organisms are able to form stable surface associated microbial communities (biofilms), which have been described as potent alternative to planktonic cells regarding their application as biocatalyst, especially when solvents or otherwise toxic compounds are involved in the processes [42]. The term 'biofilm' refers to surface-associated microbial communities as well as microbes forming flocks and aggregates. Biofilm organisms develop on all kinds of interfaces (e.g., oil/water/air) and are embedded in self-produced extracellular polymeric substances (EPS) in which they live in a coordinate fashion, thereby benefitting from ecological niches formed within the biofilm [43]. Biofilmgrowing organisms are self-regenerating, spatially and metabolically well organized, and are in general less affected by toxic substrates and/ or products [43]. Accordingly the Pseudomonas sp. can be considered as exceptional biocatalysts and can accelerate bioremediation when other species are not fit. (Figure 1) Venn diagram showing unique and shared OTUs (97\%) in each sample ENGA and ENGB and the total richness for all groups by pyrosequencing. Figure Relative abundance of the microbial community before the inoculation of the enriched consortium (ENGA) and after 40 days of bioremediation of the creosote contaminated soil (ENG B).

\section{Identification of biosurfactant-producing isolates}

Biosurfactant-producing microorganisms are ubiquitous, inhabiting both water (sea, fresh water, groundwater) and land (soil, sediment, sludge) as well as extreme environments (e.g. Oil reservoirs), and thriving at a wide range of temperatures, $\mathrm{pH}$ values and salinity. They can also be isolated from undisturbed environments where they have physiological roles not involving the solubilisation of hydrophobic pollutants e.g., antimicrobial activity, biofilm formation or processes of motility and colonization of surfaces [44]. However, it is among the hydrocarbon-degrading microbial communities that the capability to produce biosurfactants is most widespread [45]. Microorganisms have adopted different strategies to enhance the bioavailability and gain access to hydrophobic compounds, such as hydrocarbons, including (1) biosurfactant mediated solubilisation, (2) direct access of oil drops and (3) biofilm-mediated access [46]. The production of biosurfactants and bioemulsifiers is generally involved, although to different degrees, in all the above strategies [45]. Biosurfactant structural uniqueness resides in the coexistence of a hydrophilic (a sugar or peptide) and a hydrophobic (fatty acid chain) domain in the same molecule, which allows them to occupy the interface of mixed phase systems (e.g., oil/ water, air/water, oil/solid/water) and consequently to alter the forces governing the equilibrium conditions. This is the prerequisite for a broad range of surface activities to take place including emulsification, dispersion, dissolution, solubilisation, wetting and foaming [45,47].

The current study suggests that the predominant OTUs identified are ubiquitous in petroleum hydrocarbon contaminated soil and were proved efficient Creosote degrading with efficient biosurfactant production capabilities. The present work shows the potential use of these microorganisms and the total consortium for the bioremediation of crude oil and petroleum polluted environmental media.

\section{Acknowledgement}

This research was funded by the National Research Foundation (NRF) of South Africa through the Incentive Funding for Rated Researchers Grant No. IFR2010042900080awarded to Prof. Evans M.N.Chirwa of the University of Pretoria.We thank Prof Fanus Venter of the Department of Microbiology and Plant Pathology, University of Pretoria, for his assistance with the DNA sequencing and characterisation of bacteria from the soil.

\section{References}

1. Xu N, Wang W, Han P, Lu X (2009) Effects of ultrasound on oily sludge deoiling Journal of hazardous materials 171: 914-917.

2. Hu G, Li J, Zeng G (2013) Recent development in the treatment of oily sludge from petroleum industry: A review. Journal of hazardous materials 261: 470490.

3. Short JW, Lindeberg MR, Harris PM, Maslko J, Rice SD (2002) Vertical oil distribution within intertidal zone 12 years after the Exxon Valdez oil spill in Prince William Sound, Alaska. Proceedings of the 25th Arctic and Marine Oilspill Program (AMOP) Technical seminar, Calgary, Alberta pp: 57-72.

4. United Press International (1986) Gasoline Reported threatening water. Boston Globe.

5. Onwurah INE, Ogugua VN, Onyike NB, Ochonogor AE, Otitoju OF (2007) Crude oil spills in the environment, Effects and Some Innovative Clean-up Biotechnologies. International Journal of Environmental Research 1: 307-320.

6. Cerqueira VS, Maria do Carmo RP, Camargo FA, Bento FM (2014) Comparison of bioremediation strategies for soil impacted with petrochemical oily sludge. International Biodeterioration and Biodegradation 95: 338-345.

7. Atlas RM, Hazen TC (2011) Oil Biodegradation and Bioremediation: A Tale of the Two Worst Spills in U.S. History. Environmental science and technology 45: 6709-6715.

8. Fuentes S, Méndez V, Aguila P, Seeger M (2014) Bioremediation of petroleum hydrocarbons: catabolic genes, microbial communities, and applications. Applied microbiology and biotechnology 98: 4781-4794.

9. Das R, Kazy SK (2014) Microbial diversity, community composition and metabolic potential in hydrocarbon contaminated oily sludge: prospects for in situ bioremediation. Environmental Science and Pollution Research 21: 73697389.

10. Diaz-Ramirez IJ, Escalante-Espinosa E, Favela-Torres E, Gutiérrez-Rojas M, Ramírez-Saad H (2008) Design of bacterial defined mixed cultures for biodegradation of specific crude oil fractions, using population dynamics analysis by DGGE. International Biodeterioration \& Biodegradation 62: 21-30.

11. Atlas RM, Bartha R (1998) Microbial Ecology Fundamentals and Applications (4thedn.) Benjamin Cummings

12. Al-Wasify RS, Hamed SR (2014) Bacterial Biodegradation of Crude Oil using Local Isolates. International Journal of Bacteriology. 
Citation: Chirwa EMN, Bezza FA (2015) Petroleum Hydrocarbon Spills in the Environment and Abundance of Microbial Community Capable of Biosurfactant Production. J Pet Environ Biotechnol 6: 237. doi:10.4172/2157-7463.1000237

Page 5 of 5

13. Mohan SV, Reddy BP, Sarma PN (2009) Ex situ slurry phase bioremediation of chrysene contaminated soil with the function of metabolic function Process evaluation by data enveloping analysis (DEA) and Taguchi design of experimental methodology (DOE). Bioresource technology 100: 164-172.

14. Mohan SV, Chandrasekhar K (2011) Self-induced bio-potential and graphite electron accepting conditions enhances petroleum sludge degradation in bioelectrochemical system with simultaneous power generation. Bioresource Technology 102: 9532-9541.

15. Belcher RW, Huynh KV, Hoang TV, Crowley DE (2012) Isolation of biosurfactant-producing bacteria from the Rancho La Brea Tar Pits. World Journal of Microbiology and Biotechnology 28: 3261-3267.

16. Brito EM, Guyoneaud R, Goñi-Urriza M, Ranchou-Peyruse A, Verbaere A, et al. (2006) Characterization of hydrocarbonoclastic bacterial communities from mangrove sediments in Guanabara Bay, Brazil. Research in microbiology 157: 752-762.

17. Albers CN, Ellegaard-Jensen L, Harder CB, Rosendahl S, Knudsen BE, etal. (2014) Groundwater chemistry determines the prokaryotic community structure of waterworks sand filters. Environmental Science and Technology 49: 839846 .

18. Trummler K, Effenberger F, Syldatk C (2003) An integrated microbial/enzymatic process for production of rhamnolipids and L-(+)-rhamnose from rapeseed oil with Pseudomonas sp. DSM 2874. European journal of lipid science and technology 105: 563-571.

19. Cookson JT (1995) Bioremediation engineering: design and application. Food and Agricultural Organization of the United Nations.

20. Sundberg C, Al-Soud WA, Larsson M, Alm E, Yekta SS, et al. (2013) 454 pyrosequencing analyses of bacterial and archaeal richness in 21 full-scale biogas digesters. FEMS microbiology ecology 85: 612-626.

21. Yu Y, Lee C, Kim J, Hwang S (2005) Group-specific primer and probe sets to detect methanogenic communities using quantitative real-time polymerase chain reaction. Biotechnology and Bioengineering 89: 670-679.

22. Cole JR, Wang Q, Cardenas E, Fish J, Chai B, et al. (2009) The Ribosomal Database Project: improved alignments and new tools for rRNA analysis. Nucleic acids research 37: D141-D145.

23. Schloss PD, Westcott SL, Ryabin T, Hall JR, Hartmann M, et al. (2009) Introducing mothur: open-source, platform-independent, community-supported software for describing and comparing microbial communities. Applied and environmental microbiology 75: 7537-7541.

24. Katoh K, Asimenos G, Toh H (2009) Multiple alignment of DNA sequences with MAFFT. Methods Mol Biol 537: 39-64.

25. Edgar RC, Haas BJ, Clemente JC, Quince C, Knight R (2011) UCHIME improves sensitivity and speed of chimera detection. Bioinformatics 27 2194-2200.

26. Wang Q, Garrity GM, Tiedje JM, Cole JR (2007) Naive Bayesian classifier for rapid assignment of rRNA sequences into the new bacterial taxonomy. Appl Environ Microbiol 73: 5261-5267.

27. Militon C, Boucher D, Vachelard C, Perchet G, Barra V, et al. (2010) Bacterial community changes during bioremediation of aliphatic hydrocarboncontaminated soil. FEMS Microbiology Ecology 74: 669-681.

28. Sutton NB, Maphosa F, Morillo JA, Al-Soud WA, Langenhoff AA, et al. (2013) Impact of long-term diesel contamination on soil microbial community structure. Applied and Environmental Microbiology 79: 619-630.

29. Prince RC, Gramain A, McGenity TJ (2010) Prokaryotic hydrocarbon degraders Handbook of Hydrocarbon and Lipid Microbiology 19: 1669-1692.

30. Kim JS, Crowley DE (2007) Microbial diversity in natural asphalts of the Rancho La Brea Tar Pits. Applied and environmental microbiology 73: 4579-4591.

31. Mahjoubi M, Jaouani A, Guesmi A, Amor SB, Jouini A, et al. (2013). Hydrocarbonoclastic bacteria isolated from petroleum contaminated sites in Tunisia: isolation, identification and characterization of the biotechnological potential. New Biotechnology 30: 723-733.

32. Philp JC, Bamforth SM, Singleton I, Atlas RM (2005) Environmental pollution and restoration: a role for Bioremediation. Bioremediation: Applied Microbial Solutions for Real-World Environmental Cleanup 1-48.

33. Bodour AA, Drees KP, Maier RM (2003) Distribution of biosurfactant-producing bacteria in undisturbed and contaminated arid Southwestern soils. Applied and Environmental Microbiology 69: 3280-3287.

34. Rodrigues L, Moldes A, Teixeira J, Oliveira R (2006) Kinetic study of fermentative biosurfactant production by Lactobacillus strains. Biochemical Engineering Journal 28: 109-116.

35. Maciel BM, Dias JC, Dos Santos AC, Filho RC, Fontana R, et al. (2007) Microbial surfactant activities from a petrochemical landfarm in a humid tropical region of Brazil. Canadian journal of microbiology 53: 937-943.

36. Najafi AR, Rahimpour MR, Jahanmiri AH, Roostaazad R, Arabian D, et al. (2011) Interactive optimization of biosurfactant production by Paenibacillus alvei ARN63 isolated from an Iranian oil well. Colloids and Surfaces B Biointerfaces 82: 33-39.

37. Patil SN, Aglave BA, Pethkar AV, Gaikwad VB (2012) Stenotrophomonas koreensis a novel biosurfactant producer for abatement of heavy metals from the environment. Afr J Microbiol Res 6: 5173-5178.

38. Amer R, Fattah YRA (2014)Hydrocarbonclastic marine bacteria in Mediterranean Sea, El-Max, Egypt: isolation, identification and site characterization. Jokull journal 64: 223-249.

39. Vieira PA, Vieira RB, De França FP, Cardoso VL (2007) Biodegradation of effluent contaminated with diesel fuel and gasoline. Journal of Hazardous Materials 140: 52-59.

40. Chamkha M, Trabelsi Y, Mnif S, Sayadi S (2011) Isolation and characterization of Klebsiella oxytoca strain degrading crude oil from a Tunisian off-shore oil field. Journal of basic microbiology 51: 580-589.

41. Nikolopoulou M (2013) Oil spills bioremediation in marine environment: biofilm characterization around oil droplets.

42. Lang K, Zierow J, Buehler K, Schmid A (2014) Metabolic engineering of Pseudomonas sp. strain VLB120 as platform biocatalyst for the production of isobutyric acid and other secondary metabolites. Microbial cell factories 13.

43. Halan B, Buehler K, Schmid A (2012) Biofilms as living catalysts in continuous chemical syntheses. Trends in biotechnology 30: 453-465.

44. Van Hamme JD, Singh A, Ward OP (2006) Physiological aspects: Part 1 in a series of papers devoted to surfactants in microbiology and biotechnology. Biotechnology Advances 24: 604-620.

45. Marchant R, Smyth APTJP, Banat IM (2010) 47 Production and Roles of Biosurfactants and Bioemulsifiers in Accessing Hydrophobic Substrates, pp.1502-1510.

46. Hommel RK (1990) Formation and physiological role of biosurfactants produced by hydrocarbon-utilizing microorganisms Biosurfactants in hydrocarbon utilization. Biodegradation 1(2-3): 107-119.

47. Desai JD, Banat IM (1997) Microbial production of surfactants and their commercial potential. Microbiology and Molecular biology reviews 61: 47-64 